\title{
DISCRETE INTEGRABLE EQUATIONS AND SPECIAL FUNCTIONS
}

\section{V.YU. NOVOKSHENOV}

\begin{abstract}
A generic scheme based on the matrix Riemann-Hilbert problem theory is proposed for constructing classical special functions satisfying difference equations. These functions comprise gamma- and zeta functions, as well as orthogonal polynomials with corresponding recurrence relations. We show that all difference equations are the compatibility conditions of certain Lax pair coming from the Riemann-Hilbert problem. At that, the integral representations for solutions to the classical Riemann-Hilbert problem on duality of analytic functions on a contour in the complex plane are generalized for the case of discrete measures, that is, for infinite sequences of points in the complex plane. We establish that such generalization allows one to treat a series of nonlinear difference equations integrable in the sense of solitons theory.

The solutions to the mentioned Riemann-Hilbert problems allows us to reproduce analytic properties of classical special functions described in handbooks and to describe a series of new functions pretending to be special. For instance, this is true for difference Painlevé equations. We provide the example of applying a difference second type Painlevé equation to the representation problem for a symmetric group.
\end{abstract}

Mathematics Subject Classification: 33C05, 33C12, 34M55, 34M40, 34E20, 34M60

In work [18], there was considered a scheme for describing classical special functions based on the matrix Riemann-Hilbert problem. It was shown that such functions satisfying ordinary differential equations can be represented in terms of a solution to some Riemann-Hilbert problem, that is, in terms of the problem on recovering an analytic function by its boundary values. In this way, for the corresponding differential equations, there was checked the integrability property treated in the sense of the solutions theory [1], [26]. Such treating of the integrability property as calculating of the values of a function by its global behavior means the presence of an integrable representation for this function. In fact, the method of the Riemann-Hilbert problem demonstrates the equivalency of these two definitions of the integrability [6], [15]. The functions covered by such treating of the integrability are, for instance, hypergeometric and elliptic functions. However, in the handbooks, see, for instance, [7], [14], [27], there are other special functions satisfying no differential equations. Among such functions are Gamma and zeta functions and their generalizations arising in the number theory, combinatorics and the groups representation theory. How one can extend the method of the Riemann-Hilbert problem to these special functions?

In the present paper we attempt to answer this question. The key point is that there exists a discrete equation satisfied by special functions. It turns out that these equations can be treated within the scheme of the solitons theory. Namely, for each discrete equation we provide the Lax pair of two linear equations and their compatibility condition is exactly the considered

V.Yu. Novokshenov, Discrete integrable equations and sPecial functions.

(c) Novokshenov V.Yu. 2017.

The work is financially supported by the grant of Russian Science Foundation (project no. 17-11-01004).

Submitted July 1, $201 \%$. 
discrete equation. In its turn, this Lax pair is constructed on the base of some spectral problem for matrix operators. The equations of the Lax pair are discrete in a spectral parameter and the differentiation is replaced by a difference operator or by an operator of another discrete transform. In Section 2 we shall show how these operators are naturally constructed via the solutions of an appropriate Riemann-Hilbert problem.

We should stress the specific features of the Riemann-Hilbert problem for the discrete case. Here the problem of matching boundary values on a continuous contour in the complex plane is replaced by defining the residues of a meromorphic function on a discrete set of the points. In the solitons theory, an analogue of this problem is recovering of eigenfunctions by the discrete spectrum of an operator and this is equivalent to solving equations of "dressing" chain for $N$-soliton solution [22]. In our case, a chain of nonlinear equations of such type arises from the discrete Riemann-Hilbert problem and possesses all integrability properties intrinsic to differential equations. In Section 3 this approach will be demonstrated at the example of a discrete Painlevé equation of second kind. In conclusion we consider briefly an application of this equation in the combinatorics in order to stress the fact that a "discrete Painlevé" transcendent serves as a new nonlinear special function.

\section{Riemann-Hilbert PRoblem and Lax Pairs}

Riemann-Hilbert problem on a contour. We begin with the classical Riemann-Hilbert problem, in which we choose a oriented Hölder contour $\Gamma$ in the complex plane $\lambda$, which possibly has self-intersection points and more than one connected component. On the contour $\Gamma$ we define an $N \times N$ invertible matrix $G=G(\lambda)$ called jump matrix. The Riemann-Hilbert problem defined by the pair $(\Gamma, G)$ consists in finding $N \times N$-matrix-valued function $Y(\lambda) \in \operatorname{Mat}(N, \mathbb{C})$ satisfying the conditions

1) $Y(\lambda)$ is piece-wise analytic in the domains $\lambda \in \mathbb{C} \backslash \Gamma$ and there exist its limits on the contour $\Gamma$

$$
Y_{ \pm}(\lambda)=\lim _{\substack{\lambda^{\prime} \rightarrow \lambda \\ \lambda^{\prime} \in \pm \text { side of } \mathbb{C} \backslash \Gamma}} Y\left(\lambda^{\prime}\right)
$$

$2) \operatorname{det} G(\lambda) \neq 0$ on the contour $\Gamma$ and jump condition

$$
Y_{+}(\lambda)=Y_{-}(\lambda) G(\lambda)
$$

holds.

3) $Y(\lambda)$ tends to the unit matrix $I$ at infinity $\lambda \rightarrow \infty$.

In the scalar case $N=1$ the Riemann-Hilbert 1)-3) is solved explicitly. Indeed, as $G(\lambda) \neq 0$, we can pass to the additive matching problem

$$
\ln Y_{+}(\lambda)=\ln Y_{-}(\lambda)+\ln G(\lambda) .
$$

The additive problem on a jump of the form $y_{+}(\lambda)=y_{-}(\lambda)+g(\lambda)$ with the condition $y(\lambda) \rightarrow 0$ at infinity is solved explicitly by means of the Cauchy integral

$$
y(\lambda)=\frac{1}{2 \pi i} \int_{\Gamma} \frac{g(\mu)}{\mu-\lambda} d \mu .
$$

Condition 2 is implied by the Sokhotski-Plemelj formula on the boundary values of the Cauchy integral (see, for instance, [11]). Moreover, Riemann-Hilbert problem 1)-3) has an explicit solution in the Abelian case, when $G\left(\lambda_{1}\right) G\left(\lambda_{2}\right)=G\left(\lambda_{2}\right) G\left(\lambda_{1}\right)$ for all $\lambda_{1}$ and $\lambda_{2}$ on the contour 
$\Gamma$. This solution is given by the formula

$$
Y(\lambda)=\exp \left\{\frac{1}{2 \pi i} \int_{\Gamma} \frac{\ln G(\mu)}{\mu-\lambda} d \mu\right\} .
$$

Thus, formula (1) is true if the jump matrix has the zero index, that is, as $\left.\Delta \ln G\right|_{\Gamma}=0$. If the index is non-zero, formula (1) is modified by multiplying by a polynomial of degree not exceeding the index [11]. In this case the Riemann-Hilbert problem is not uniquely solvable and has finitely many linearly independent solutions.

In the non-Abelian case as $N>1$, when the matrices $G\left(\lambda_{1}\right)$ and $G\left(\lambda_{2}\right)$ do not commute on the contour $\Gamma$, the formula (1) is not applicable. As a rule, in this case there is not explicit solution for the Riemann-Hilbert problem. Nevertheless, the theorems on unique solvability are true for wide classes of jump matrices. For instance, a sufficient condition for the unique solvability of the Riemann-Hilbert problem is the positive definiteness of the matrix $G(\lambda)$ [4]. In the general case, the Riemann-Hilbert problem 1)-3) is reduced to solving a system of singular integral equations for the entries of the matrix $Y(\lambda)$ [11. This approach is useful not only for proving the solvability, but also for estimating the norm of the matrix $Y(\lambda)$ and for analyzing its asymptotic behavior.

In the applications, the jump matrix depend on the additional parameters. Here we restrict ourselves by the case $N=2$ and by one scalar parameter $x$ and

$$
G(\lambda, x)=\mathrm{e}^{p(\lambda, x) \sigma_{3}} S \mathrm{e}^{-p(\lambda, x) \sigma_{3}}, \quad \sigma_{3}=\left(\begin{array}{cc}
1 & 0 \\
0 & -1
\end{array}\right),
$$

where $S$ is a constant in $\lambda$ and $x$ matrix, and $p$ is a scalar polynomial in $\lambda$ and $x$. Then the solution of Riemann-Hilbert problem 1)-3) also depends on the paramerer $x$. Introducing new matrices $\Psi(\lambda, x)=Y(\lambda, x) \mathrm{e}^{p(\lambda, x) \sigma_{3}}$, we obtain the Riemann-Hilbert problem

$\left.1^{\prime}\right) \Psi(\lambda, x)$ is piecewise analytic in $\lambda$ as $\lambda \in \mathbb{C} \backslash \Gamma$

$\left.2^{\prime}\right) \Psi_{+}(\lambda, x)=\Psi_{-}(\lambda, x) S, \quad \lambda \in \Gamma$.

$\left.3^{\prime}\right) \Psi(\lambda, x) \rightarrow \mathrm{e}^{p(\lambda, x) \sigma_{3}}, \quad \lambda \rightarrow \infty$.

We consider the logarithmic derivatives:

$$
A(\lambda, x)=\Psi_{\lambda} \Psi^{-1}, \quad U(\lambda, x)=\Psi_{x} \Psi^{-1} .
$$

Condition $2^{\prime}$ ) implies ( $S$ is a constant matrix!):

$$
\begin{aligned}
& A_{+}(\lambda, x)=\left(\Psi_{-}\right)_{\lambda} S \Psi_{+}^{-1}=\left(\Psi_{-}\right)_{\lambda} \Psi_{-}^{-1}=A_{-}(\lambda, x), \\
& U_{+}(\lambda, x)=\left(\Psi_{-}\right)_{x} S \Psi_{+}^{-1}=\left(\Psi_{-}\right)_{x} \Psi_{-}^{-1}=U_{-}(\lambda, x), \quad \lambda \in \Gamma .
\end{aligned}
$$

Therefore, $A$ and $U$ are analytic in $\lambda$ in the entire complex plane. On the other hand, it follows from Condition $3^{\prime}$ ) that at infinity, these matrices has a polynomial growth:

$$
A(\lambda, x) \rightarrow p_{\lambda}(\lambda, x) \sigma_{3}, \quad U(\lambda, x) \rightarrow p_{x}(\lambda, x) \sigma_{3}, \quad \lambda \rightarrow \infty,
$$

By the Liouville theorem, the matrices $A$ and $U$ are matrix polynomials in $\lambda$ of the degree $\operatorname{deg} p_{\lambda}$ and $\operatorname{deg} p_{x}$, respectively. Thus, the matrix $\Psi(\lambda, x)$ is determined by an over-determined system of differential equations with polynomial coefficients (Lax pair):

$$
\left\{\begin{array}{l}
\Psi_{\lambda}=A \Psi \\
\Psi_{x}=U \Psi
\end{array}\right.
$$


The compatibility condition of system (5) is the equation

$$
A_{x}-U_{\lambda}+[A, U]=0 .
$$

This equation is separated into a finite set of matrix equations for the coefficients at each power of $\lambda$ and its turn, this gives equations for scalar functions of $x$. Under a proper choice of the pair $(\Gamma, G)$, in this way we can get differential equations for the special functions and their integral representations.

For instance, choosing the contour $\Gamma$ as the union of three rays, $\Gamma_{k}=\{\lambda \mid \arg \varkappa=\pi / 2+2 \pi k / 3\}$, $k=0,1,2$, letting $p(\lambda, x)=8 \lambda^{3} / 3+\lambda x$ and defining the matrix $S$ in formula (2) as [9, Ch. 3],

$$
S_{k}=\left(\begin{array}{cc}
1 & s_{k} \\
0 & 1
\end{array}\right), \quad s_{0}+s_{1}+s_{2}=0
$$

we obtain an explicit solution to Riemann-Hilbert problem 1)-3) as

$$
Y(\lambda, x)=\left(\begin{array}{cc}
1 & y(\lambda, x) \\
0 & 1
\end{array}\right), \quad y(\lambda, x)=\left\{s_{0} \int_{\Gamma_{0}}+s_{1} \int_{\Gamma_{1}}+s_{2} \int_{\Gamma_{2}}\right\} \frac{\mathrm{e}^{p(\mu, x)}}{\mu-\lambda} d \mu .
$$

At that, equation (6) is equivalent to the Airy equation $u^{\prime \prime}=x u$ for the function

$$
u(x)=-\lim _{\lambda \rightarrow \infty} \lambda y(\lambda, x)=\left\{s_{0} \int_{\Gamma_{0}}+s_{1} \int_{\Gamma_{1}}+s_{2} \int_{\Gamma_{2}}\right\} \mathrm{e}^{p(\mu, x)} d \mu .
$$

In Section 2 we shall provide the examples of employing Riemann-Hilbert problem 1)-3) for other special functions.

Disctere Riemann-Hilbert problem. Nonlinear difference equations for special functions requires another version of the Riemann-Hilbert problem. Following works by A.Borodin [2], [3], we define a discrete Riemann-Hilbert problem as follows.

Let $\Sigma$ be some countable set of points in the complex plane $\lambda \in \mathbb{C}$ with the only accumulation point at infinity. Let $H(\lambda)$ be a matrix function on $\Sigma, H: \Sigma \rightarrow \operatorname{Mat}(N, \mathbb{C})$.

We shall say that the matrix-valued function $Y: \mathbb{C} \backslash \Sigma \rightarrow \operatorname{Mat}(N, \mathbb{C})$ with simple poles at the points $x \in \Sigma$ is a solution to the discrete Riemann-Hilbert problem $(\Sigma, H)$ if the following conditions hold:

$1^{\circ} Y(\lambda)$ is analytic in $\mathbb{C} \backslash \Sigma$ and has simple poles at the points $\Sigma$,

$$
2^{\circ} \operatorname{Res}_{\lambda=x} Y(\lambda)=\lim _{\lambda \rightarrow x}(Y(\lambda) H(x)), x \in \Sigma,
$$

$3^{\circ} Y(\lambda) \rightarrow I$ as $\lambda \rightarrow \infty$.

As above, $H(\lambda)$ is called the jump matrix.

We note that this formulation of the discrete Riemann-Hilbert problem is very similar to the pure soliton case in inverse scattering problem [26, Part III].

We denote

$$
\mathbb{Z}^{\prime}=\mathbb{Z}+\frac{1}{2}=\left\{\ldots,-\frac{3}{2},-\frac{1}{2}, \frac{1}{2}, \frac{3}{2}, \ldots\right\}=\mathbb{Z}_{+}^{\prime} \cup \mathbb{Z}_{-}^{\prime},
$$

where $\mathbb{Z}_{+}^{\prime}=\left\{\frac{1}{2}, \frac{3}{2}, \ldots\right\}$ and $\mathbb{Z}_{-}^{\prime}=\left\{\ldots,-\frac{3}{2},-\frac{1}{2}\right\}$. 
Let us consider the construction of the Lax pair for problem $1^{\circ}-3^{\circ}$ in the particular case $N=2$

$$
\begin{aligned}
& \Sigma_{k}=\{k, k+1, k+2, \ldots\}, \quad k \in \mathbb{Z}^{\prime}, \\
& H(x)= \begin{cases}\left(\begin{array}{cc}
\left.0-\frac{\varkappa^{2 x}}{\Gamma^{2}\left(x+\frac{1}{2}\right)}\right), & x \in \mathbb{Z}_{+}^{\prime}, \\
0 & 0
\end{array}\right) \\
\left(\begin{array}{cc}
0 & 0 \\
-\frac{\varkappa^{-2 x}}{\Gamma^{2}\left(-x+\frac{1}{2}\right)} & 0
\end{array}\right), & x \in \mathbb{Z}_{-}^{\prime} .\end{cases}
\end{aligned}
$$

It was proved in paper [2] that there exists the unique solution to problem $\left(\Sigma_{k}, H\right)$. Following [3], let us prove that for each $n \in \mathbb{Z}_{k}$ there exists a constant nilpotent matrix $A_{n}$,

$$
A_{n}=\left(\begin{array}{cc}
p_{n} & q_{n} \\
r_{n} & -p_{n}
\end{array}\right), \quad p_{n}^{2}=-r_{n} q_{n}
$$

and functions $a_{n}, b_{n}, a_{n} b_{n}=1$, such that

$$
\begin{aligned}
& Y_{n+1}(\lambda)=\left(I+\frac{A_{n}}{\lambda-n}\right) Y_{n}(\lambda), \\
& Y_{n}(\lambda-1)\left(\begin{array}{cc}
\varkappa^{-1}\left(\lambda-\frac{1}{2}\right) & 0 \\
0 & \varkappa\left(\lambda-\frac{1}{2}\right)^{-1}
\end{array}\right)=\left(\begin{array}{cc}
\varkappa^{-1}\left(\lambda-\frac{1}{2}-p_{n}\right) & a_{n} \\
-b_{n} & 0
\end{array}\right) Y_{n+1}(\lambda) .
\end{aligned}
$$

Indeed, since $H$ is independent of $n$, we see that $Y_{n}(\lambda)$ and $Y_{n+1}(\lambda)$ satisfy the same condition at $\Sigma_{n}$. However, $Y_{n+1}$ has an extra pole at zero $\{n\}=\Sigma_{n+1} \backslash \Sigma_{n}$. Therefore, the quotient $Y_{n+1} Y_{n}^{-1}$ has one pole at the point $\lambda=n$. Denoting the residue at this point by $A_{n}$, we conclude that the function

$$
Y_{n+1}(\lambda) Y_{n}^{-1}(\lambda)-\frac{A_{n}}{\lambda-n}
$$

is entire. Calculating the asymptotics in the vicinity of $\lambda=\infty$, by the Liouville theorem we obtain that this function is identically equal to $I$ that proves the first equation. It follows from $\operatorname{det} Y_{n} \equiv \operatorname{det} Y_{n+1} \equiv 1$ that $\operatorname{det}\left(I+A_{n} /(\lambda-n)\right) \equiv 1$. By this we conclude that $A_{n}$ is nilpotent.

We observe that Condition $2^{\circ}$ means that the function $Y(\lambda)$ has an essential singularity at infinity. Indeed, the function with the poles accumulating at infinity can not have a regular asymptotics. In order to make the condition well-posed, we should, for instance, assume an uniform asymptotics at the sequence of circumferences $|\lambda|=a_{k}, a_{k} \rightarrow+\infty$.

In order to ensure the uniqueness of solution to the discrete Riemann-Hilbert problem considered below in Section 3, we shall assume that there exists the sequence of expanding contours separated by a positive distance from $\Sigma$ and we shall assume that the solution $Y(\lambda)$ possesses a required asymptotics at these contours.

In view of these remarks, let us calculate the asymptotics of $Y_{n}(\lambda)$ at infinite. Condition $3^{\circ}$ implies that

$$
Y_{n}(\lambda)=I+\left(\begin{array}{ll}
\alpha_{n} & \beta_{n} \\
\gamma_{n} & \delta_{n}
\end{array}\right) \lambda^{-1}+O\left(\lambda^{-2}\right), \quad \lambda \rightarrow \infty,
$$

with some constants $\alpha_{n}, \ldots, \delta_{n}$.

To get equation (10), we divide it at the left by $Y_{n+1}(\lambda)$ and let us prove that its left hand side

$$
Y_{n}(\lambda-1)\left(\begin{array}{cc}
\varkappa^{-1}\left(\lambda-\frac{1}{2}\right) & 0 \\
0 & \varkappa\left(\lambda-\frac{1}{2}\right)^{-1}
\end{array}\right) Y_{n+1}^{-1}(\lambda)
$$

is a polynomial in $\lambda$. 
By (11), the asymptotics of matrix (12) is of the form

$$
\begin{aligned}
& \left.\left(I+\left(\begin{array}{ll}
\alpha_{n} & \beta_{n} \\
\gamma_{n} & \delta_{n}
\end{array}\right) \lambda^{-1}\right)\left(\begin{array}{cc}
\varkappa^{-1}\left(\lambda-\frac{1}{2}\right) & 0 \\
0 & 0
\end{array}\right)\left(I-\left(\begin{array}{ll}
\alpha_{n+1} & \beta_{n+1} \\
\gamma_{n+1} & \delta_{n+1}
\end{array}\right) \lambda^{-1}\right)\right)+O\left(\lambda^{-1}\right) \\
& =\varkappa^{-1}\left(\begin{array}{cc}
\lambda-\frac{1}{2}+\alpha_{n}-\alpha_{n+1} & -\beta_{n+1} \\
\gamma_{n} & 0
\end{array}\right)+O\left(\lambda^{-1}\right) .
\end{aligned}
$$

We denote $a_{n}=-\varkappa^{-1} \beta_{n+1}, b_{n}=-\varkappa^{-1} \gamma_{n}, c_{n}=\alpha_{n+1}-\alpha_{n}$. Then it follows from Liouville theorem that expression (12) is equal to

$$
\left(\begin{array}{cc}
\varkappa^{-1}\left(\lambda-\frac{1}{2}-c_{n}\right) & a_{n} \\
-b_{n} & 0
\end{array}\right)
$$

In conclusion let us show that $c_{n}=p_{n}$ and $a_{n} b_{n}=1$. The second identity is implied by the fact that the determinant of $Y_{n}(\lambda)$ is equal to 1 . In order to prove that $c_{n}=p_{n}$, we substitute (9) into just proved relation $(10)$. We get:

$$
\begin{aligned}
Y_{n}(\lambda-1) & \left(\begin{array}{cc}
\varkappa^{-1}\left(\lambda-\frac{1}{2}\right) & 0 \\
0 & \varkappa\left(\lambda-\frac{1}{2}\right)^{-1}
\end{array}\right) \\
= & \left(\begin{array}{cc}
\varkappa^{-1}\left(\lambda-\frac{1}{2}-c_{n}\right) & a_{n} \\
-b_{n} & 0
\end{array}\right)\left(I+(\lambda-n)^{-1}\left(\begin{array}{cc}
p_{n} & q_{n} \\
r_{n} & -p_{n}
\end{array}\right)\right) Y_{n}(\lambda) .
\end{aligned}
$$

Comparing the asymptotics of the matrix entries $(\cdot)_{11}$ in this identity, we conclude that $c_{n}=p_{n}$. This proves the equations in Lax pair (9) and (10).

\section{LINEAR DIFFERENCE EQUATIONS}

As it has been mentioned in Introduction, there are many special functions satisfying no differential equations. In this case, what does serve as the integrability property for such functions? We are going to demonstrate this property at three examples.

Gamma function. We define a "cut" Gamma function as follows:

$$
\gamma(x) \stackrel{\text { def }}{=} \frac{1}{2 \pi i}\left(1-\mathrm{e}^{2 \pi i x}\right) \Gamma(x+1)=\frac{1}{2 \pi i} \int_{C} \mathrm{e}^{-\theta(\lambda, x)} d \lambda,
$$

where $\theta(\lambda, x)=\lambda-x \ln \lambda$ and the integration contour $C$ envelops the positive semi-axis and this contour is passed clockwise. We define the Abelian Riemann-Hilbert problem as $N=2$ on the contour $C$ with the jump matrix [16]

$$
G(\lambda, x)=\left(\begin{array}{cc}
1 & \mathrm{e}^{-\theta(\lambda, x)} \\
0 & 1
\end{array}\right)
$$

Its solution is reduced to two scalar problems of matching analytic functions and its solution is given by the explicit formula

$$
Y(\lambda, x)=\left(\begin{array}{cc}
1 & \int_{C} \frac{\mathrm{e}^{-\theta(\mu, x)}}{\mu-\lambda} d \mu \\
0 & 1
\end{array}\right)
$$

The corresponding Lax pair is written in terms of $\Psi$-function (see [16])

$$
\begin{aligned}
& \Psi(\lambda, x)=Y(\lambda, x) \mathrm{e}^{-\theta(\lambda, x) \sigma_{3} / 2} \lambda^{\frac{x}{2} \sigma_{3}} \\
& A=\Psi_{\lambda}(\lambda, x) \Psi^{-1}(\lambda, x)=-\frac{\sigma_{3}}{2}+\frac{1}{\lambda}\left(\begin{array}{cc}
x / 2 & -\gamma(x) \\
0 & -x / 2
\end{array}\right), \\
& U=\Psi(\lambda, x+1) \Psi^{-1}(\lambda, x)=-\sqrt{\lambda}\left(\begin{array}{cc}
0 & \gamma(x) \\
0 & 1
\end{array}\right)+\frac{1}{\sqrt{\lambda}}\left(\begin{array}{cc}
0 & \gamma(x) \\
0 & 1
\end{array}\right),
\end{aligned}
$$


where the compatibility condition is of the form

$$
U_{\lambda}=A(\lambda, x+1) U-U A(\lambda, x) .
$$

The latter identity splits into the chain of identities and one non-trivial difference equation

$$
\gamma(x+1)=(x+1) \gamma(x),
$$

which implies the determining equation for the Gamma function:

$$
\Gamma(x+1)=x \Gamma(x) .
$$

Riemann zeta function. As above, we begin with the integral representation for $\zeta$-function 27]

$$
\pi^{-\frac{x}{2}} \Gamma\left(\frac{x}{2}\right) \zeta(x)=-\frac{1}{x}+\frac{1}{x-1}+\int_{0}^{1} \omega\left(\lambda^{-1}\right) \lambda^{\frac{x}{2}-\frac{3}{2}} d \lambda+\int_{1}^{\infty} \omega(\lambda) \lambda^{\frac{x}{2}-1} d \lambda
$$

where

$$
\omega(\lambda)=\sum_{n=1}^{\infty} \mathrm{e}^{-\pi n^{2} \lambda} \equiv \frac{1}{2}\left(\theta_{3}(0 \mid i \lambda)-1\right)
$$

and

$$
\theta_{3}(z \mid \kappa)=\sum_{m} \mathrm{e}^{\pi i \kappa m^{2}+2 \pi i z m}
$$

is the Jacobi theta function of the variable $x$ with the modulus $\kappa$ [7].

The Riemann relation for $\zeta$-function

$$
\pi^{-\frac{x}{2}} \Gamma\left(\frac{x}{2}\right) \zeta(x)=\pi^{-\frac{1-x}{2}} \Gamma\left(\frac{1-x}{2}\right) \zeta(1-x)
$$

is rewritten in terms of the function

$$
\xi(x)=\int_{0}^{1} \omega\left(\lambda^{-1}\right) \lambda^{\frac{x}{2}-\frac{3}{2}} d \lambda+\int_{1}^{\infty} \omega(\lambda) \lambda^{\frac{x}{2}-1} d \lambda
$$

as the difference equation

$$
\xi(x)=\xi(1-x), \quad x \neq 0, \quad x \neq 1 .
$$

Following [15], we define the contour $C$ as the union of the segments $\Gamma_{1}=[0,1]$ and $\Gamma_{2}=[1,+\infty)$ with the natural orientation and choose the jump matrix as

$$
G(\lambda, x)=\left\{\begin{array}{cl}
\left(\begin{array}{cc}
1 & 2 \pi i \omega\left(\lambda^{-1}\right) \lambda^{\frac{x}{2}-\frac{3}{2}} \\
0 & 1
\end{array}\right), & \lambda \in \Gamma_{1}, \\
\left(\begin{array}{cc}
1 & 2 \pi i \omega(\lambda) \lambda^{\frac{x}{2}-1} \\
0 & 1
\end{array}\right), & \lambda \in \Gamma_{2} .
\end{array}\right.
$$

By the Abelian property of the Riemann-Hilbert problem $\left(\Gamma_{1} \cup \Gamma_{2}, G\right)$, its solution $Y(\lambda, x)$ exists and is unique. In order to obtain the corresponding Lax pair, we define the $\Psi$-function as follows:

$$
\Psi(\lambda, x)=Y(\lambda, x) \lambda^{\left(\frac{x}{4}-\frac{5}{8}\right) \sigma_{3}}, \quad \sigma_{3}=\left(\begin{array}{cc}
1 & 0 \\
0 & -1
\end{array}\right)
$$

Then the matrix $\Psi$ satisfies the equations:

$$
\left\{\begin{array}{l}
\sigma_{3} \Psi\left(\frac{1}{\lambda}, 5-x\right) \sigma_{3}=A(\lambda, x) \Psi(\lambda, x) \\
\Psi(\lambda, s+2)=U(\lambda, x) \Psi(\lambda, x)
\end{array}\right.
$$


where the matrices $A$ and $U$ are of the form:

$$
A(\lambda, x)=\left(\begin{array}{cc}
1 & -\xi(3-x) \\
0 & 1
\end{array}\right), \quad U(\lambda, x)=\left(\begin{array}{cc}
\frac{1}{\sqrt{\lambda}} & -\xi(x) \\
0 & \sqrt{\lambda}
\end{array}\right) .
$$

The equations of Lax pair (15) are implied by the estimate of "logarithmic derivatives"

$$
\sigma_{3} \Psi\left(\frac{1}{\lambda}, 5-x\right) \sigma_{3} \Psi^{-1}(\lambda, x) \text { and } \Psi(\lambda, x+2) \Psi^{-1}(\lambda, x) .
$$

In their turn, the latter estimates are implied by the invariance of jump matrices (14) w.r.t. the shift $x \mapsto x+2$ and modular transform of theta-constant $\theta_{3}(0 \mid i / \lambda)=\sqrt{\lambda} \theta_{3}(0 \mid i \lambda)$ (see [7], [15]). The compatibility condition for Lax pair (15) is

$$
\sigma_{3} U\left(\frac{1}{\lambda}, 3-x\right) \sigma_{3} A(\lambda, x+2) U(\lambda, x) A^{-1}(\lambda, x)=I,
$$

which is equivalent to difference equation 13 .

Orthogonal polynomials. As a contour $C$, we choose the real axis with the natural orientation $C=\mathbb{R}$. The jump matrix is chosen as

$$
G(\lambda)=\left(\begin{array}{cc}
1 & 2 \pi i w(\lambda) \\
0 & 1
\end{array}\right), \quad \lambda \in \Gamma
$$

where

$$
w(\lambda)=\mathrm{e}^{-V(\lambda)}, \quad V(\lambda)=\sum_{j=1}^{2 k} \lambda^{j} t_{j}, \quad t_{2 k}>0 .
$$

We consider the Riemann-Hilbert problem $(\Gamma, G)$, in which Condition 3) is replaced by

$$
Y(\lambda)\left(\begin{array}{cc}
\lambda^{-n} & 0 \\
0 & \lambda^{n}
\end{array}\right) \rightarrow I, \quad \lambda \rightarrow \infty, \quad n \in \mathbb{Z}_{+} .
$$

It was proved in work by A.S. Fokas, A.R. Its, and A.V. Kitaev [8] that this problem possesses the unique solution for each $n \in \mathbb{Z}_{+}$. Moreover, this solution can be represented as

$$
Y_{n}(\lambda)=\left(\begin{array}{cc}
P_{n}(\lambda) & \int_{-\infty}^{\infty} \frac{P_{n}(\mu) w(\mu) d \mu}{\lambda-\mu} \\
\frac{1}{h_{n-1}} P_{n-1}(\lambda) & \frac{1}{h_{n-1}} \int_{-\infty}^{\infty} \frac{P_{n-1}(\mu) w(\mu) d \mu}{\lambda-\mu}
\end{array}\right),
$$

where $\left\{P_{n}\right\}_{n=1}^{\infty}$ is the family of the polynomials of the form

$$
P_{n}(\lambda)=\lambda^{n}+a_{n, n-1} \lambda^{n-1}+\ldots+a_{n, 0} .
$$

It is easy to find the properties of these polynomials by the formulation of the Riemann-Hilbert problem. In particular, they are orthogonal on the real axis with the weight $w(\lambda)=\mathrm{e}^{-V(\lambda)}$ :

$$
\int_{-\infty}^{\infty} P_{n}(\lambda) P_{m}(\lambda) w(\lambda) d \lambda=h_{n} \delta_{n m} .
$$

In order to prove formula 19 we write out the asymptotics at infinity by condition (17)

$$
Y_{n}(\lambda)=\left\{I+m_{1}^{(n)} \lambda^{-1}+O\left(\lambda^{-2}\right)\right\}\left(\begin{array}{cc}
\lambda^{n} & 0 \\
0 & \lambda^{-n}
\end{array}\right), \quad \lambda \rightarrow \infty
$$


and hence,

$$
\begin{aligned}
& \left(Y_{n}(\lambda)_{22}=\lambda^{-n}+O\left(\lambda^{-n-1}\right)=\frac{1}{h_{n-1}} \int_{-\infty}^{\infty} \frac{P_{n-1}(\mu) w(\mu) d \mu}{\lambda-\mu}=\right. \\
& =\frac{1}{\lambda h_{n-1}} \int_{-\infty}^{\infty} P_{n-1}(\mu) w(\mu)\left(1+\frac{\mu}{\lambda}+\ldots+\frac{\mu^{n}}{\lambda^{n}}+\ldots\right) d \mu .
\end{aligned}
$$

This implies

$$
h_{n-1}=\int_{-\infty}^{\infty} P_{n-1}(\mu) w(\mu) \mu^{n-1} d \mu, \quad 0=\int_{-\infty}^{\infty} P_{n-1}(\mu) w(\mu) \mu^{k} d \mu, \quad 0 \leqslant k \leqslant n-2,
$$

which proves identity (19).

The orthogonal polynomials satisfy a linear recurrent identity relating polynomials with indices $n-1, n$ and $n+1$ [25]. It can be considered as an equation in $n$ for the family of the polynomials orthogonal with a given weight.

In order to get this recurrent identity, we consider the "logarithmic derivative"

$$
U_{n}(\lambda)=Y_{n+1}(\lambda) Y_{n}^{-1}(\lambda)
$$

It is obvious that by explicit formulae (16) and (18), the function $U_{n}(z)$ is analytic in the entire complex plane. Let us find the asymptotics for $U_{n}(\lambda)$ at infinity. By asymptotics (20), the matrix $U_{n}$ has the following asymptotic expansion

$$
U_{n}(\lambda)=Y_{n+1}(\lambda) Y_{n}^{-1}(\lambda)=\lambda\left(\begin{array}{ll}
1 & 0 \\
0 & 0
\end{array}\right)+m_{1}^{(n+1)}\left(\begin{array}{ll}
1 & 0 \\
0 & 0
\end{array}\right)-\left(\begin{array}{ll}
1 & 0 \\
0 & 0
\end{array}\right) m_{1}^{(n)}+\ldots
$$

Thus, as $\lambda \rightarrow \infty$,

$$
Y_{n+1} Y_{n}^{-1}=\left(\begin{array}{cc}
\lambda & 0 \\
0 & 0
\end{array}\right)+\left(\begin{array}{cc}
a_{n+1}-a_{n} & -b_{n} \\
c_{n+1} & 0
\end{array}\right)+O\left(\lambda^{-1}\right), \quad \text { where } \quad m_{1}^{(n)}=\left(\begin{array}{ll}
a_{n} & b_{n} \\
c_{n} & d_{n}
\end{array}\right) .
$$

By the Liouville theorem

$$
U_{n}(\lambda)=\left(\begin{array}{cc}
\lambda+a_{n+1}-a_{n} & -b_{n} \\
c_{n+1} & 0
\end{array}\right)
$$

which gives the difference equation for the entry $(\cdot)_{11}$ :

$$
P_{n+1}(\lambda)=\lambda P_{n}(\lambda)+\left(a_{n+1}-a_{n}\right) P_{n}(\lambda)-\frac{b_{n}}{h_{n-1}} P_{n-1}(\lambda) .
$$

Finally, the recurrent identity is of the form

$$
P_{n+1}(\lambda)+\left(\alpha_{n}-\lambda\right) P_{n}(\lambda)+\beta_{n} P_{n-1}(\lambda)=0,
$$

where

$$
\begin{aligned}
& \alpha_{n}=a_{n}-a_{n+1}=\lim _{\lambda \rightarrow \infty}\left[\lambda^{1-n}\left(Y_{n}(\lambda)\right)_{11}-\lambda^{-n}\left(Y_{n+1}(\lambda)\right)_{11}\right], \\
& \beta_{n}=\frac{b_{n}}{h_{n-1}}=\lim _{\lambda \rightarrow \infty} \lambda^{2}\left(Y_{n}(\lambda)\right)_{12}\left(Y_{n}(\lambda)\right)_{21} .
\end{aligned}
$$

In the particular case of the weight function $w(\lambda)=\mathrm{e}^{-\lambda^{2}}$ we get the Hermit polynomials (see [5, Ch. 3]):

$$
P_{n}(\lambda)=H_{n}(\lambda)=(-1)^{n} e^{\lambda^{2}} \frac{d^{n}}{d \lambda^{n}} e^{-\lambda^{2}} .
$$


In this case the Riemann-Hilbert problem allows us to calculate and justify the asymptotics for the Hermit polynomial as $n \rightarrow \infty$, see [5, Ch. 7]. This calculation reproduces PlancherelRotach formulae [21], at that, the method of "asymptotic undressing" of the Riemann-Hilbert problem in [6] is applicable for other classes of polynomials [19].

In conclusion we note that in the case of exponential weight $(16)$ with the parameters $t=$ $\left\{t_{1}, \ldots, t_{2 k}\right\}$, the coefficients $\alpha_{n}$ and $\beta_{n}$ in recurrent identity 21) become functions of $t$. At that, they satisfy integrable equations. For instance, in the case

$$
V(\lambda)=\frac{\lambda^{4}}{4}+t \lambda^{2}
$$

the coefficient $\beta_{n}$ satisfies Painlevé equation of fourth type

$$
\left.u_{t t}=\frac{u_{t}^{2}}{2 u}+\frac{1}{2 u}\left(3 u^{2}+2 t u-n-1\right)\right)\left(u^{2}+2 t u+n+1\right),
$$

where $u(t)=\beta_{n} h_{n-1}[17]$.

\section{NONLINEAR DIFFERENCE EQUATIONS}

Equation dPII. We apply the discrete Riemann-Hilbert problem considered in Section 1 to obtain a discrete analogue of the second Painlevé equation (dPII). The solutions of the classical differential Painlevé equation are by right among "nonlinear" special functions (see [9]) thanks to numerous applications in various problems of mathematics and physics. Below we shall show that the solutions to the discrete equation dPII also deserve a status of special functions.

Following work [2] and the discrete Riemann-Hilbert problem $1^{\circ}-3^{\circ}$ formulate in Section 1 on the set $\Sigma_{k}$ with jump matrix (7), we are going to obtain the compatibility conditions for Lax pair (9), (10). Shifting $\lambda$ to 1 in $(10)$ and substituting the right hand side (10) into the right hand side in (9), we obtain

$$
Y_{n+1}(\lambda)=\left(I+\frac{A_{n}}{\lambda-n}\right)\left(\begin{array}{cc}
\varkappa^{-1}\left(\lambda+\frac{1}{2}-p_{n}\right) & a_{n} \\
-b_{n} & 0
\end{array}\right) Y_{n+1}(\lambda+1)\left(\begin{array}{cc}
\varkappa\left(\lambda+\frac{1}{2}\right)^{-1} & 0 \\
0 & \varkappa^{-1}\left(\lambda+\frac{1}{2}\right)
\end{array}\right) .
$$

On the other hand, shifting $n$ and $\lambda$ to 1 in (9) and $(10)$ and substituting the right hand side of (9) into the right hand side in (10), we obtain

$Y_{n+1}(\lambda)=\left(\begin{array}{cc}\varkappa^{-1}\left(\lambda+\frac{1}{2}-p_{n+1}\right) & a_{n+1} \\ -b_{n+1} & 0\end{array}\right)\left(I+\frac{A_{n+1}}{\lambda-n}\right) Y_{n+1}(\lambda+1)\left(\begin{array}{cc}\varkappa\left(\lambda+\frac{1}{2}\right)^{-1} & 0 \\ 0 & \varkappa^{-1}\left(\lambda+\frac{1}{2}\right)\end{array}\right)$.

Comparing these two relations, we obtain the compatibility condition for Lax pair (9), (10):

$$
\left(I+\frac{A_{n}}{\lambda-n}\right)\left(\begin{array}{cc}
\varkappa^{-1}\left(\lambda+\frac{1}{2}-p_{n}\right) & a_{n} \\
-b_{n} & 0
\end{array}\right)=\left(\begin{array}{cc}
\varkappa^{-1}\left(\lambda+\frac{1}{2}-p_{n+1}\right) & a_{n+1} \\
-b_{n+1} & 0
\end{array}\right)\left(I+\frac{A_{n+1}}{\lambda-n}\right) .
$$

This relation is the analogue of equation (6) obtained by cross differentiation of the equation in the Lax pair in Section 1.

By the matrix equation (22), one can get easily scalar equations for the variables $p_{n}$ and $r_{n}$. Namely, calculating the asymptotics of the entries $(\cdot)_{12}$ and $(\cdot)_{21}$ in identity (22) as $\lambda \rightarrow \infty$ yields the relations

$$
\left\{\begin{array}{l}
a_{n}=a_{n+1}+\varkappa^{-1} q_{n+1}, \quad b_{n}=b_{n+1}+\varkappa^{-1} r_{n} \\
a_{n} r_{n}=-b_{n+1} q_{n+1} .
\end{array}\right.
$$

The residues at a simple pole $\lambda=n$ in identity (22) are of the form

$$
\left(\begin{array}{cc}
p_{n} & q_{n} \\
r_{n} & -p_{n}
\end{array}\right)\left(\begin{array}{cc}
\varkappa^{-1}\left(n+\frac{1}{2}-p_{n}\right) & a_{n} \\
-b_{n} & 0
\end{array}\right)=\left(\begin{array}{cc}
\varkappa^{-1}\left(n+\frac{1}{2}-p_{n+1}\right) & a_{n+1} \\
-b_{n+1} & 0
\end{array}\right)\left(\begin{array}{cc}
p_{n+1} & q_{n+1} \\
r_{n+1} & -p_{n+1}
\end{array}\right) .
$$


The matrix entry $(\cdot)_{22}$ of this identity coincides with the last identity in $(23)$, while the entry $(\cdot)_{12}$ gives

$$
a_{n} p_{n}=\varkappa^{-1}\left(\lambda+\frac{1}{2}-p_{n+1}\right) q_{n+1}-a_{n+1} p_{n+1} .
$$

Multiplying both sides by $b_{n+1}$, we obtain (recall that $a_{n+1} b_{n+1}=1$ )

$$
b_{n+1} a_{n} p_{n}=-\varkappa^{-1}\left(\lambda+\frac{1}{2}-p_{n+1}\right) a_{n} r_{n}-p_{n+1} .
$$

We denote

$$
s_{n}=a_{n} r_{n},
$$

and multiplying the first identity in $(23)$ by $b_{n+1}$, we see that $a_{n} b_{n+1}=1-\varkappa^{-1} s_{n}$. Substituting this expression into (24), we obtain:

$$
\left(p_{n}+p_{n+1}\right)\left(s_{n}-\varkappa\right)=\left(n+\frac{1}{2}\right) s_{n} .
$$

Employing the nilpotent property of the matrix $A_{n}$ (8), we have

$$
p_{n+1}^{2}=-q_{n+1} r_{n+1}=\left(-b_{n+1} q_{n+1}\right)\left(a_{n+1} r_{n+1}\right)=s_{n} s_{n+1} .
$$

Thus, for each $n \in \mathbb{Z}_{k}$ we obtain the system of scalar equations

$$
\left\{\begin{array}{l}
\left(p_{n}+p_{n+1}\right)\left(s_{n}-\varkappa\right)=\left(n+\frac{1}{2}\right) s_{n} \\
p_{n+1}^{2}=s_{n} s_{n+1}
\end{array}\right.
$$

We can exclude easily the variable $p_{n}$ from this system, namely, letting

$$
s_{n}=\varkappa x_{n}^{2},
$$

we obtain the scalar difference equation

$$
x_{n+1}+x_{n-1}=\frac{\left(n+\frac{1}{2}\right) x_{n}}{\varkappa\left(x_{n}^{2}-1\right)} .
$$

Equation (25) is equation dPII or difference Painlevé II equation, (see [3], [13], [24]). Let us show [20] that this equation becomes the differential Painlevé II equation in the limit $\varkappa \rightarrow \infty$. We introduce a continuous variable $t$ as

$$
t=(n-2 \varkappa) \varkappa^{-\frac{1}{3}}
$$

and assume that $x_{n} \approx(-1)^{n} \varkappa^{-\frac{1}{3}} u(t)$ as $\varkappa \rightarrow \infty$ with some smooth function $u(\cdot)$. Then

$$
\begin{aligned}
& x_{n \pm 1}=(-1)^{s+1} \varkappa^{-\frac{1}{3}}\left(u(t) \pm \varkappa^{-\frac{1}{3}} u^{\prime}(t)+\varkappa^{-\frac{2}{3}} u^{\prime \prime}(t)+O\left(\varkappa^{-1}\right)\right), \\
& \frac{\left(n+\frac{1}{2}\right) x_{n}}{\varkappa\left(x_{n}^{2}-1\right)}=(-1)^{n+1}\left(2+\varkappa^{-\frac{2}{3}} t+\frac{1}{2} \varkappa^{-1}\right) \varkappa^{-\frac{1}{3}} u(t)\left(1+\varkappa^{-\frac{2}{3}} u^{2}(t)+O\left(\varkappa^{-\frac{4}{3}}\right)\right) \\
& =(-1)^{n+1} \varkappa^{-\frac{1}{3}}\left(2 u(t)+\varkappa^{-\frac{2}{3}}\left(t u(t)+2 u^{3}(t)\right)+O\left(\varkappa^{-1}\right)\right) .
\end{aligned}
$$

Substituting this into 25 and passing to the limit as $\varkappa \rightarrow \infty$, we obtain

$$
u^{\prime \prime}(t)=t u(t)+2 u^{3}(t)
$$

which a particular case of Painlevé II equation [9]. 
Representation of symmetric group. Let $S_{n}$ be a symmetric group of degree $n$, that is, the group of permutations of a set with $n$ elements denoted usually by the natural numbers $1,2, \ldots, n$. We denote by $l_{n}(\sigma)$ the length of the maximal increasing sequence of a permutation $\sigma \in S_{n}$ and by $|\cdot|$ we denote the number of the elements in a set. we let

$$
p_{k}^{n}=\frac{1}{n !}\left|\left\{\sigma \in S_{n} \mid l_{n}(\sigma) \leqslant k\right\}\right|,
$$

and introduce the generating function

$$
p_{k}(\varkappa)=\mathrm{e}^{-\varkappa^{2}} \sum_{n=0}^{\infty} \frac{\varkappa^{2 n}}{n !} p_{k}^{n},
$$

where $\varkappa$ is some complex parameter. Another equivalent definition follows the RobinsonSchensted correspondence [10]. We take all partitions of the permutation $\lambda=\left(\lambda_{1}, \lambda_{2}, \ldots, \lambda_{l}\right) \in$ $S_{|\lambda|}$ such that $\left|\lambda_{1}\right| \geqslant \ldots \geqslant\left|\lambda_{l}\right|>0,\left|\lambda_{1}\right| \leqslant k$ and $|\lambda|=\left|\lambda_{1}\right|+\cdots+\left|\lambda_{l}\right|$. We denote by $\operatorname{dim} \lambda$ the dimension of a irreducible symmetric group $S_{|\lambda|}$, then

$$
p_{k}(\varkappa)=\mathrm{e}^{-\varkappa^{2}} \sum_{\left|\lambda_{1}\right| \leqslant k}\left(\frac{\operatorname{dim} \lambda}{|\lambda| !} \varkappa^{|\lambda|}\right)^{2},
$$

where the summation is taken over all such partitions $\lambda$.

The calculation of the function $p_{k}(\varkappa)$ is an important issue in the representation theory of symmetric group. It was proved in work [12] that this function can be written as the Toeplitz determinant

$$
p_{k}(\varkappa)=\mathrm{e}^{-\varkappa^{2}} \operatorname{det}\left[f_{i-j}\right]_{i, j=1}^{k}, \quad \sum_{m=-\infty}^{+\infty} f_{m} \zeta^{m}=\mathrm{e}^{\varkappa\left(\zeta+\zeta^{-1}\right)} .
$$

In paper [23] there was first established a relation between the function $p_{k}(\varkappa)$ and a solution to equation dPII. We define a sequence $\left\{x_{n}\right\}_{n=0}^{\infty}$ by the initial conditions $x_{0}=-1, x_{1}=f_{1} / f_{0}$ with $f_{i}$ in 26 ) and by the recurrent relation

$$
x_{n+1}+x_{n-1}=\frac{n x_{n}}{\varkappa\left(x_{n}^{2}-1\right)}, \quad n \geqslant 1 .
$$

Then in the general situation, for each $k \geqslant 1$ and $\varkappa$, the recurrent relations

$$
\frac{p_{k+1}(\varkappa) p_{k-1}(\varkappa)}{p_{k}^{2}(\varkappa)}=1-x_{k}^{2}
$$

hold true. Here the words "in the general situation" mean that $\varkappa$ does not belong to the set of the poles of the meromorphic function $x_{k}=x_{k}(\varkappa)$.

Another way of obtaining this results by means of the discrete Riemann-Hilbert problem was given later in [2].

\section{BIBLIOGRAPHY}

1. M.J. Ablowitz and P.A. Clarkson Solitons, nonlinear evolution equations and inverse scattering. Math. Soc. Lecture Notes Series. 149. Cambridge Univ. Press, Cambridge (1991).

2. A. Borodin. Discrete gap probabilities and discrete Painlevé equations // Duke Math. J. 117:3, $1-54(2003)$.

3. A. Borodin. Isomonodromy transformations of linear systems of difference equations // Ann. Math. 160:3, 1141-1182 (2004).

4. K. Clancey and I. Gohberg. Factorization of matrix functions and singular integral operators. Operator Theory: Advances and Applications. 3. Birkhauser Verlag, Basel (1981).

5. P. Deift. Orthogonal polynomials and random matrices: A Riemann-Hilbert approach. Courant Lecture Notes. New York Univ. New York (1999). 
6. P.A. Deift. Integrable Systems and Combinatorial Theory // Notices Amer. Math. Soc. 47:6, 631-640 (2000).

7. A. Erdelyi, W. Magnus, F. Oberhettinger and F. G. Tricomi. Tables of integral transforms. V. I. McGraw-Hill Book Company, Inc., New York (1954).

8. A.S. Fokas, A.R. Its, and A.V. Kitaev. The isomonodromy approach to matrix models in $2 D$ quantum gravity // Comm. Math. Phys. 147:2, 395-430 (1992).

9. A.S. Fokas, A.R. Its, A.A. Kapaev and V.Yu. Novokshenov. Painlevé transcendents. The Riemann-Hilbert approach. Math. Surveys and Monographs. 128. Amer. Math. Soc., Providence, RI (2006).

10. W. Fulton. Young tableaux: with applications to representation theory and geometry. London Math. Soc. Cambridge Univ. Press, Cambridge (1997).

11. F.D. Gakhov. Boundary value problems. Inter. Ser. Monog. Pure Appl. Math. 85. Pergamon Press, Oxford (1990).

12. I.M. Gessel. Symmetric functions and P-recursiveness // J. Combin. Theory. Ser. A. 53:2, 257-285 (1990).

13. B. Grammaticos, F. W. Nijhoff, A. Ramani. Discrete Painlevé equations // in "The Painlevé property. One century later." ed. R. Conte. CRM Ser. Math. Phys. Springer, New York, 413-516 (1999).

14. E.L. Ince. Ordinary differential equations. Dover, New York (1956).

15. A.R. Its The Riemann-Hilbert problem and integrable systems // Notices Amer. Math. Soc. 50:11, 1389-1400 (2003).

16. A.V. Kitaev. Special functions of the isomonodromy type // Acta Appl. Math. 64:1, 1-32 (2000).

17. A.P. Magnus. Painlevé-type differential equations for the recurrence coefficients of semi-classical orthogonal polynomials // J. Comput. Appl. Math. 57:1-2, 215-237 (1995).

18. V.Yu. Novokshenov. The Riemann-Hilbert problem and special functions // In: "Geometric Methods in Physics". AIP Conf. Proc. 1079:1, 149-161 (2008).

19. V.Yu. Novokshenov, A.A. Schelkonogov. Distribution of zeroes to generalized Hermite polynomials // Ufimskij Matem. Zhurn. 7:3, 57-69 (2015).

20. Y. Ohta, A. Ramani, B. Grammaticos, K. M. Tamizhmani. From discrete to continuous Painlevé equations: a bilinear approach // Phys. Lett. A. 216:6, 255-261 (1996).

21. M. Plancherel, W. Rotach. Sur les valeurs asymptotiques des polynomes d'Hermite $H_{n}(x)=$ $(-I)^{n} e^{\frac{x^{2}}{2}} \frac{d^{n}}{d x^{n}}\left(e^{-\frac{x^{2}}{2}}\right) / /$ Commentarii Math. Helvetici. 1:1, 227-254 (1929).

22. A.B. Shabat. The infinite-dimensional dressing dynamical system // Inverse Probl. 8:2, 303-308 (1992).

23. C.A. Tracy and H. Widom. Random unitary matrices, permutations and Painlevé // Comm. Math. Phys. 207:3, 665-685 (1999).

24. H. Sakai. Rational surfaces associated with affine root systems and geometry of the Painlevé equations // Comm. Math. Phys. 220:1, 165-229 (2001).

25. G. Szegö. Orthogonal polynomials. Colloquium Publ. 23. Amer. Math. Soc., New York (1959).

26. S. Novikov, S.V. Manakov, L.P. Pitaevskii, V.E. Zakharov. Theory of solitons. The inverse scattering method. Nauka, Moscow (1980). [Contem. Soviet Math. Plenum Publishing Corp., New York (1984).]

27. E.T. Whittaker and G.N. Watson. A Course of modern analysis. Cambridge Univ. Press, Cambride (1996).

Viktor Yurievich Novokshenov,

Institute of Mathematics, Ufa Scientific Center, RAS,

Chernyshevsky str. 112,

450008, Ufa, Russia

E-mail: novik530mail.ru 